\title{
Reducing neuronal apoptosis in pontine micturition center by nerve root transfer to reconstruct bladder function after spinal cord injury
}

\section{Ronghua Yu}

Tongren Hospital Shanghai Jiaotong University School of Medicine

\section{Gang Yin}

shanghai general hospital

Jianguo Zhao

changzeng hospital

Huihao Chen

Changzheng Hospital

Depeng Meng

Changzheng Hospital

Jiaqiang Zhang

Changzheng Hospital

Yaofa Lin

Changzheng Hospital

Zheng Xie

Changzheng Hospital

Chunlin Hou

Changzheng Hospital

Haodong Lin ( $\square$ haodonglin@hotmail.com )

https://orcid.org/0000-0003-3461-3430

\section{Research article}

Keywords: neuronal apoptosis, spinal cord injury, nerve root transfer, bladder function reconstruction

Posted Date: November 18th, 2019

DOI: https://doi.org/10.21203/rs.2.17382/v1

License: (a) This work is licensed under a Creative Commons Attribution 4.0 International License.

Read Full License 


\section{Abstract}

Background: The neuronal apoptosis is increased after spinal cord injury (SCl), but anastomosing the normal nerve roots above $\mathrm{SCl}$ level to the injury sacral nerve roots can enhance functional recovery of neurons. Therefore, we evaluated the effect of sacral nerve root transfer after $\mathrm{SCl}$ on pontine neuronal survival and restoration of bladder function.

Methods: Adult female Sprague Dawley rats ( $N=90,9-10$ weeks old, 240-260 grams weight) were randomly divided into three groups $(\mathrm{N}=30)$. We anastomosed the dorsal and ventral roots of proximal $\mathrm{L} 4$ and distal S2 to reconstruct the rat bladder-spinal cord-cerebral nerve afferent and efferent pathways in Sprague Dawley rats after spinal cord transection. We examined pontine neuronal morphology and apoptosis using hematoxylin and eosin (H\&E) staining and transmission electron microscopy (TEM) at different time points (1 day, 1 week, and 1, 3, or 6 months) after SCl and nerve transfer. Bcl-2 and Bax protein expression changes in the pontine micturition center were quantified by immunohistochemistry.

Results: After nerve roots reconstruction, Group A compared with Group B, Bcl-2 expression increased significantly, Bax expression decreased significantly, Bcl-2/Bax ratio increased, the number of apoptotic neurons decreased, and the number of apoptotic bodies within neurons decreased significantly as observed by TEM.

Conclusion: These findings demonstrate that lumbosacral nerve transfer can reduce neuronal apoptosis in the pontine micturition center and enhance functional recovery of neurons. This method can be used as a new approach for reconstructing bladder function after spinal cord injury.

\section{Introduction}

After $\mathrm{SCl}$, atrophy appears in the corresponding functional areas of the brain as neurons gradually disappear and become fibrotic, or replaced by surrounding functional areas [1,2]. Animal experiments and clinical cases have demonstrated that anastomosing the anterior and posterior nerve roots above the injury level to the anterior and posterior sacral nerve roots controlling the bladder can simultaneously reconstruct the afferent and efferent pathways of the bladder and improve bladder function [3-6]. However, the structural changes and the mechanisms underlying functional remodeling of the micturition center in the brain after reconstruction of the bladder afferent and efferent pathways remain unclear. In the present study, anastomosis of the dorsal and ventral roots of the nerves above the injury level and the dorsal and ventral sacral nerve roots controlling the bladder was used to reconstruct the rat bladderspinal cord-cerebral nerve afferent and efferent pathways. $\mathrm{Bcl}-2$ and Bax protein expression changes were examined in the pontine micturition center to explore the role of neuronal apoptosis at various times after spinal cord injury and nerve transfer. Our findings demonstrate that lumbosacral nerve transfer can reduce neuronal apoptosis in the pontine micturition center and enhance functional recovery of micturition. 


\section{Materials And Methods}

\section{Animal models and experimental groups}

Adult female Sprague Dawley rats ( $N=90,9-10$ weeks old, 240-260 grams weight) were purchased from the Experimental Animal Center of the Second Military Medical University. All rats were raised and tested in the Experimental Animal Center of the Second Military Medical University. They were housed at a constant temperature of $23 \pm 2{ }^{\circ} \mathrm{C}$, humidity $55 \pm 5 \%$, free diet, and 12 -h light/dark cycle. The rats were randomly divided into three groups, with 30 rats in each of the following groups: Group A, reconstruction of afferent and efferent nerve pathways of the bladder performed after SCl; Group B, SCl only; and Group C, control group.

Rats were anesthetized with an intraperitoneal injection of $1 \%$ sodium pentobarbital at $40 \mathrm{mg} / \mathrm{kg}$. The conus medullaris was exposed at level of L4 (Fig. 1a), and the spinal cord was cut below L4. In Group A, the bilateral $\mathrm{L} 4$ nerve roots were isolated from the endorhachis and cut at the intervertebral foramen. The bilateral S2 nerve roots were dissected and cut. 12-0 microsutures were used for tension-free suturing of the dorsal and ventral roots of bilateral S2 and L4 (Fig. 1b, C).. In Group B, the severed spinal cord was not treated. In Group C, the spinal cord and spinal nerves below $L 4$ were exposed but not lesioned. Following surgery, the rats were intraperitoneally injected with gentamicin $(0.5 \mathrm{mg} / \mathrm{kg})$ once daily for 3 days.

Abdominal massage was performed twice daily (in the morning and evening) to help expel residual urine. The rats were sacrificed by intraperitoneal injection of $1 \%$ sodium pentobarbital solution at 1 day, 1 week, and 1, 3, or 6 months after surgery, 6 in each, and the dorsolateral pontine tegmentum was dissected. Bladder tissues were dissected at 3 months after surgery. Each group took at least five rats each time.

\section{Morphological analysis}

The dorsolateral pontine tegmentum and bladder tissues were post-fixed with $4 \%$ paraformaldehyde, washed with normal saline, and dehydrated with increasing concentrations of ethanol. Longitudinal sections $5 \mu \mathrm{m}$ thick were sliced and retained for routine hematoxylin and eosin (H\&E) staining. Ten visual fields were randomly selected, and structural changes in the dorsolateral pontine tegmentum and bladder tissues were observed under a light microscope.

\section{Electrophysiological studies of the bladder}

Three months after surgery, the rats in each group were anesthetized with $1 \%$ pentobarbital, the bladder was emptied, and a $20 \mathrm{G}$ intravenous indwelling blunt needle was inserted into the external urethral orifice. The intravenous indwelling needle was connected with a micro-injection pump and pressure transducer, respectively, via a three-way tube, and the pressure transducer was connected to a physiological signal device (RM6240C Chengdu Instrument Factory). Intravesical instillation of saline at $37^{\circ} \mathrm{C}$ at a speed of 9 $\mathrm{ml} / \mathrm{h}$ was performed to measure the pressure-volume curve. The S1 and S2 nerve roots of the rats were 
then dissected. The stimulus electrodes were placed at the distal end of the anastomosis of the dorsal roots of S2 and the posterior roots of the normal S1 nerve roots. Changes in bladder internal pressure were recorded. The stimulus intensity was $3 \mathrm{~mA}$, the pulse width was $0.3 \mathrm{~ms}$, the stimulus frequency was $20 \mathrm{~Hz}$, and the duration was $5 \mathrm{~s}$.

\section{Immunohistochemistry for $\mathrm{Bcl}-2$ and Bax proteins}

Tissue samples from the dorsolateral pontine tegmentum underwent routine deparaffinization, hydration, antigen recovery, and washing in phosphate-buffered saline (PBS). Normal goat serum blocking solution was added followed by the addition of Bcl-2 or Bax mouse monoclonal antibodies $₫$ Anti-Bcl-2 antibody (ab194583)

Description: Rabbit polyclonal to Bcl-2,Application:ICC/IF, IHC-P, WB, Reactivity: Mouse, Rat, Human, Conjugate: Unconjugated. Anti-Bax antibody (ab32503), Description: Rabbit monoclonal to Bax, Application: IHC-P, IP, sELISA, WB, Reactivity: Mouse, Rat, Human, Chinese hamster (predicted: Cow),

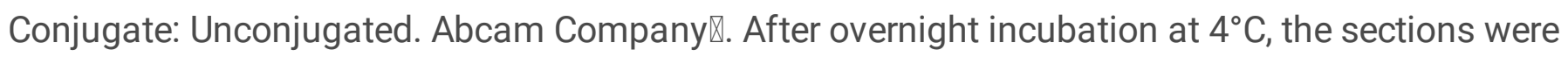
rewarmed at $37^{\circ} \mathrm{C}$ for $45 \mathrm{~min}$. The sections were then washed in PBS three times for 5 min each. A secondary horseradish peroxidase-labeled goat anti-mouse antibody was added dropwise and incubated with the tissue at $37^{\circ} \mathrm{C}$ for $30 \mathrm{~min}$. 3,3'-diaminobenzidine development was conducted for 5-10 min. After washing in PBS for $5 \mathrm{~min}$, the tissue was counterstained with hematoxylin for $2 \mathrm{~min}$ and differentiated with hydrochloric acid and ethanol. The tissue was washed in PBS for 5-10 min, dehydrated, and cleared before being coverslip ped. The sections were incubated at $180^{\circ} \mathrm{C}$ in sodium citrate solution $(\mathrm{H} 3300, \mathrm{pH}$ 6.0) for $15 \mathrm{~min}$. The slides were cooled down during $20 \mathrm{~min}$ and they were washed two times with distilled water. Five visual fields were randomly selected from each section under an inverted fluorescence microscope. ImageJ software (National Institutes of Health, Bethesda, MD) was used for analysis. Immunopositive cells were considered those with brown particles present in the cytoplasm. The proportion of the number of immunopositive cells (brown cytoplasm) to the total number of cells in a field was obtained, and a mean value was calculated for each section.

\section{Apoptotic cell count}

Samples from dorsolateral pontine tegmentum were deparaffinized and terminal deoxynucleotidyl transferase dUTP nick end labeling (TUNEL) staining was conducted. The sections were deparaffinized and rehydrated, rinsed in $0.1 \mathrm{M}$ PBS for 10 minutes, and then reacted with proteinase $\mathrm{K}(20 \mu \mathrm{g} / \mathrm{mL})$ for 30 minutes at $37^{\circ} \mathrm{C}$. After washing in PBS, the sections were incubated with equilibration buffer $(200 \mathrm{mM}$ potassium cacodylate, $25 \mathrm{mM}$ Tris- $\mathrm{HCl}, 0.2 \mathrm{mM}$ DTT, $0.2 \mathrm{mg} / \mathrm{mL}$ goat serum albumin, for 30 minutes at room temperature. The specimens were then incubated in labeling reaction mix (TdT and dUTP) for 1 hour at room temperature in the dark. The sections were then incubated with $2 \times$ saline citrate $(300 \mathrm{mM}$ $\mathrm{NaCl}, 30 \mathrm{mM}$ sodium citrate) for 10 minutes at $25^{\circ} \mathrm{C}$, washed in PBS, and reacted with DAPI for 5 minutes at room temperature in the dark. Fluorescence microscopy (Olympus) was used to acquire images. The 
positive control was produced by adding DNasel for 10 minutes at $25^{\circ} \mathrm{C}$. dUTP was used for the negative control. Samples were observed under an inverted fluorescence microscope, and five visual fields were randomly selected from each section to determine the number of apoptotic cells.

\section{Transmission electron microscopy (TEM)}

Samples from dorsolateral pontine tegmentum measuring less than $1 \mathrm{~mm}^{3}$ in volume were cut, fixed with $2.5 \%$ glutaraldehyde, dehydrated with increasing concentrations of ethanol, embedded in paraffin, sectioned to 50-60 nm with an ultrathin microtome, stained with $3 \%$ uranyl acetate-lead citrate, and observed by TEM.

\section{Statistical analysis}

Statistical analyses were performed using SPSS 19.0 Statistical Software (SPSS). Comparisons between groups were analyzed by two-way analysis of variance. $P<0.05$ was considered statistically significant. Data are expressed as mean \pm standard deviation.

\section{Results}

A total of three rats in Group A died at 1, 3, and 5 months after surgery, and a total of three rats in Group B died during the first week and at 3 and 4 months after surgery. The causes of death were thought to be wound infection or renal failure. All rats in Group C survived.

\section{Bladder function recovery}

The bladder mucosa from rats in Group A (spinal cord lesion plus nerve root anastomosis) appeared smooth and the mucosal cell arrangement was regular. The muscle layer was slightly thickened, and smooth muscle cells were slightly hypertrophic and arranged regularly. In rats from Group B (spinal cord lesion alone), the bladder mucosa was rough and the mucosal cells appeared disordered. The muscle layer was thickened and hypertrophic with fibrous tissue proliferation and inflammatory cell infiltration, and smooth muscle cells showed disordered arrangement. In rats from Group C (control), the bladder mucosa was smooth and the mucosal cells were organized in a regular pattern. The muscle layer was thin, and the smooth muscle cells were small and arranged regularly (Fig. 2a)..

The electrophysiological results from rats in Groups A and C at 3 months after surgery showed after electrical stimulation the intravesical pressure fluctuated drastically, and the urine was discharged. The intravesical pressure did not fluctuate in Group B (Fig. 2b)..

\section{Morphological analysis}


H\&E staining showed that the pontine neurons in rats from Group C were dense and arranged neatly, with clear nucleoli. One week after surgery, the extracellular space around the neurons was slightly increased in Groups A and B compared with that in Group C, and one month after surgery, some neurons showed nuclear pyknosis in Groups A and B. There was no significant difference in tissue morphology between Groups A and B when examined on the first week, and first month after surgery. Three months after surgery, an accumulation of interstitial fibrous tissue was evident. The number of neurons increased in Group A, but increasingly more cells in Group B displayed nuclear pyknosis, with disappearance of the nucleus (Fig. 3).. This suggests that the apoptosis rate of neurons after nerve root transfer (Group A) was lower than that without nerve root transfer (Group B) at three and six months after surgery.

\section{Immunohistochemistry for Bcl-2 and Bax protein expression}

Neuronal Bcl-2 expression increased gradually in Group A, and reached the highest level one week after surgery. The expression of $\mathrm{Bcl}-2$ increased again gradually one month after surgery. In contrast, the expression of $\mathrm{Bcl}-2$ in Group $\mathrm{B}$ decreased gradually over time. The intensity of $\mathrm{Bcl}-2$ expression in the dorsolateral pontine tegmentum in Group A was significantly higher than Group B at 3 and 6 months after surgery $(P<0.05)$. The expression of $\mathrm{Bcl}-2$ in Group $\mathrm{C}$ was minimally changed or unchanged at each time point. Statistically significant differences in $\mathrm{Bcl}-2$ expression were observed between Group $\mathrm{C}$ and the other groups at each time point $(P<0.05)($ Fig. $4 a)$..

The mean expression of Bax gradually increased in Group A, reaching the highest level one week after injury, followed by a gradual decrease in expression over time. Bax expression gradually increased again one month after injury. Compared with Group $B$, the intensity of Bax expression in the dorsolateral pontine tegmentum in Group A was significantly lower at 3 and 6 months after surgery $(P<0.05)$. Bax expression in Group $\mathrm{C}$ was minimally changed or unchanged at each time point. Statistically significant differences in Bax expression were observed between Group $C$ and the other groups at each time point $(P<0.01)($ Fig. $4 b)$.

The mean ratio of $\mathrm{Bcl}-2 / \mathrm{Bax}$ in Group $\mathrm{A}$ gradually increased. The mean ratio of $\mathrm{Bcl}-2 / \mathrm{Bax}$ in Group $\mathrm{B}$, however, increased gradually before one month and then gradually decreased. The $\mathrm{Bcl}-2 / \mathrm{Bax}$ ratio in Group $\mathrm{C}$ was minimally changed or unchanged at each time point. Statistically significant differences in $\mathrm{Bcl}-2 / \mathrm{Bax}$ ratio were observed between Group $\mathrm{C}$ and the other groups at each time point $(P<0.05)$. Compared with Group B, the ratio of $\mathrm{Bcl}-2 /$ Bax expression in Group A was significantly higher at three and six months after surgery $(P<0.01)($ Fig. $4 C)$..

\section{Number of apoptotic neurons}

The number of apoptotic neurons in Group A peaked at one week and then decreased gradually. The number of apoptotic neurons in Group B also peaked at one week and then decreased, but there was no 
significant change in the number of apoptotic neurons. Compared with Group B, the mean number of apoptotic neurons in Group A was significantly lower at three and six months after surgery $(P<0.05)$. The number of apoptotic neurons in Group $\mathrm{C}$ was minimally changed or unchanged at each time point. Statistically significant differences in the number of apoptotic neurons were observed between Group $\mathrm{C}$ and the other groups at one day and one week as well as one and three months after surgery $(P<0.05)$ (Fig. 4d)..

\section{TEM analysis}

TEM analysis showed nuclear pyknosis and mitochondrial vacuolization in Groups A and B at one month after surgery, as well as pyknotic margination of chromatin, intercellular space enlargement, and apoptotic bodies. The number of apoptotic cells increased in Group B. At 6 months after surgery, the neurons in Group A appeared plump, with an increase in organelle size and substantially more mitochondria compared to earlier time points (Fig. 5)..

\section{Discussion}

Recent studies have shown that damage to the mammalian nervous system can cause changes in functional plasticity of the brain [7, 8]. A study by Fraser et al. [9] found that changes in the brain sensory cortex after central nervous system injury can lead to functional remodeling of the cerebral motor cortex. Many subsequent studies [10-13]have confirmed that plasticity changes occur in the brain after SCl. However, functional remodeling of the brain after $\mathrm{SCl}$ does not always occur. Some corresponding brain functional zones undergo apoptosis, necrosis, degeneration, invasion by surrounding functional areas, or fibrosis after $\mathrm{SCl}$ [14-16]. Experiments by Jure et al. [17] determined that the occurrence of brain degeneration or neuronal apoptosis is associated with the degree of $\mathrm{SCl}$. Severe $\mathrm{SCl}$ causes apoptosis of hippocampal neurons in rats, whereas mild SCl does not cause obvious neuronal damage.

Thus, researchers have started to study whether functional remodeling after $\mathrm{SCl}$ is also related to the degree of SCl. A study by Shoham et al. [18] indicated that cerebral motor cortex activity in patients with $\mathrm{SCl}$ after remodeling is closely linked to the undamaged brain sensory cortex, suggesting an important role for the sensory cortex in brain remodeling. Jurkiewicz et al. [19] were the first to report plasticity changes in the sensorimotor cortex at various times during recovery from incomplete $\mathrm{SCl}$. They found that extensive activation of sensory cortical areas caused reorganization of the motor cortex and that once the reorganization was complete, the sensory cortical area activation states recovered consistent with motor cortical areas, suggesting that the sensory cortex plays an important role in motor cortex remodeling.

To confirm this, Jurkiewicz et al. [20] subsequently performed a similar study in patients with complete $\mathrm{SCl}$. After observing patients with complete $\mathrm{SCl}$ for 1 year, they found that the sensory cortex was not extensively activated and no change in functional remodeling was detected in the motor cortex, suggesting that functional remodeling of the motor cortex relies on extensive activation of the sensory 
cortex. Lotze et al. [21] performed a similar study, conducting functional magnetic resonance imaging and transcranial magnetic stimulation on six patients with complete thoracic $\mathrm{SCl}$. After complete thoracic $\mathrm{SCl}$, the cortical area representing the thorax gradually shrank and was replaced by representations of the upper limb muscles; the cortical areas representing the thorax were not later restored. These results demonstrate that brain function remodeling after $\mathrm{SCl}$ is related to the injury. For complete $\mathrm{SCl}$, the sensory cortex is not extensively activated because the brain receives no afferent nerve stimulation. However, plasticity changes in the motor cortex are dependent on the sensory cortex. Thus, for complete $\mathrm{SCl}$, only neuronal apoptosis and atrophy occur at the relevant functional brain areas. By contrast, for incomplete $\mathrm{SCl}$, nerve conduction pathways are repaired and reconstructed through residual axons and nerves. Continuous nerve conduction pathways may be a necessary condition for brain remodeling.

Recent studies $[3-6,22]$ have reported that reconstructing the conduction pathway from the spinal cord to the brain through nerve repair or transplantation can promote brain remodeling. Our urodynamic results showed that the intravesical pressure increased gradually after bladder instillation, with urination occurring at the point of urination pressure in Groups A and C. For rats in Group B (SCl only), light and electron microscopy results confirmed that neuronal apoptosis and degeneration occurred in the pontine micturition center. In Group A, cell regeneration and functional remodeling was evident in the pontine center at 3 and 6 months after surgery. We speculate that reconstruction of neural pathways, especially reconstruction of the bladder-to-cerebral afferent pathway, plays a critical role. In Group B, complete spinal cord injury led to afferent nerve block and no activation of the periaqueductal gray (PAG). Therefore, there was no functional remodeling in the pontine micturition center. However, in Group A, the afferent pathway of the bladder was reconstructed by anastomosing the anterior and posterior roots of $S 2$ and $L 4$, and the PAG could be extensively reactivated. The connection of the PAG with the cerebral cortex and Barrington's nucleus enabled the pontine micturition center to receive nerve stimulation again, which may be an important mechanism underlying its functional remodeling.

Changes in the expression of $\mathrm{Bcl}-2$ and Bax in Groups A and B were similar up to the first month after surgery. The expression of $\mathrm{Bcl}-2$ and Bax increased the day after surgery, peaked at the first week after surgery, and began to decline within the first month after surgery. However, in Group A, Bcl-2 expression was elevated again at 3 and 6 months after surgery, whereas Bax expression continued to decrease, leading to a continuous increase in the $\mathrm{Bcl}-2 / \mathrm{Bax}$ ratio. In Group $\mathrm{B}$, by contrast, $\mathrm{Bcl}-2$ continued to decline, while Bax remained unchanged, resulting in a continuous decrease in the $\mathrm{Bcl}-2 / \mathrm{Bax}$ ratio. These results led to a statistically significant difference in the $\mathrm{Bcl}-2 / \mathrm{Bax}$ ratio between the two groups. Because the $\mathrm{Bcl}-2 / \mathrm{Bax}$ ratio determines whether cells are apoptotic, this difference may be a mechanism underlying the repair and functional remodeling of nerve tissue observed in Group A and the neuronal apoptosis and degeneration observed in Group B. The mean number of apoptotic neurons in Group A at 3 and 6 months after surgery was significantly lower than that in Group B, consistent with the aforementioned results.

\section{Conclusions}


The results of the present study indicate that after complete $\mathrm{SCl}$, reconstruction of the bladder nerve conduction pathway in rats can lead to functional remodeling of the pontine micturition center. At three and six months after surgery, increased Bcl-2 expression, decreased Bax expression, and a reduced number of apoptotic neurons in the neural reconstruction group may be associated with the reconstruction of a bladder-cerebral nerve conduction pathway.

\section{Declarations}

SCl: spinal cord injury; TEM: transmission electron microscopy; H\&E: hematoxylin and eosin; PBS: phosphate-buffered saline; PAG: periaqueductal gray

\section{Acknowledgments}

Not applicable.

\section{Authors'contributions}

The idea for the manuscript was conceived by HCL and LHD. YRH, YG, ZJG, CHH, MDP, ZJQ, LYF and XZ carried out experiments; YRH, YG and ZJG performed data analysis; $Y R H$ and YG wrote the paper. All authors read and approved the final manuscript.

\section{Funding}

This work was supported by the National Natural Scientific Foundation of China (grant number 81571207).

\section{Availability of data and materials}

The datasets used and/or analyzed during the current study are available from the corresponding author on reasonable request.

\section{Ethical approval and consent to participate}

We certified that all applicable institutional and governmental regulations concerning the ethical use of animals were followed during the course of this research. The Ethics Committee of the Second Military Medical University approved this study. All efforts were made to minimize animal suffering and to reduce the number of animals used. Experiments were performed in accordance with the National Institute of Health Guide for the Care and Use of Laboratory Animals. 


\section{Consent for publication}

Not applicable.

\section{Competing interests}

The authors declare that they have no competing interests

\section{Author details}

1 Department of Orthopedic Surgery, Tongren hospital, Shanghai Jiaotong University School of Medicine, Shanghai, 200336, China

2 Department of Orthopedic Surgery, Changzheng Hospital, Second Military Medical University,Shanghai, 200003 China

3 Department of Orthopedic Surgery, Shanghai General Hospital, Shanghai Jiaotong University School of Medicine, Shanghai, 200080, China

4 Department of Orthopedic Surgery, Taikang Xianlin Drum Tower Hospital, Medical College of Nanjing University, Nanjing, 210046, China

\section{References}

1. Wu J, Zhao Z, Kumar A, Lipinski MM, Loane DJ, Stoica BA, Faden Al. Endoplasmic Reticulum Stress and Disrupted Neurogenesis in the Brain Are Associated with Cognitive Impairment and DepressiveLike Behavior after Spinal Cord Injury.J Neurotrauma. 2016;33(21):1919-1935.

2. Mikulis DJ, Jurkiewicz MT, Mcllroy WE, Staines WR, Rickards L, Kalsi-Ryan S, et al. Adaptation in the motor cortex following cervical spinal cord injury. Neurology. 2002;58(5):794-801.

3. Lin HD, Hou CL, Chen AM. Reconstructed bladder innervation above the level of spinal cord injury to produce urination by abdomen-to-bladder reflex contractions. J Neurosurg Spine. 2011;14(6):799802.

4. Lin HD, Hou CL, Chen AM, Xu Z. Innervation of reconstructed bladder above the level of spinal cord injury for inducing micturition by contractions of the abdomen-to-bladder reflex arc. Neurosurgery. 2010;66(5):948-952

5. Lin HD, Hou CL, Chen AM, Xu Z. Reinnervation of atonic bladder after conus medullaris injury using a modified nerve crossover technique in canines. J World Neurosurg . 2010;73(5):582-586.

6. Lin HD, Hou CL, Xu Z. Bypassing spinal cord injury: surgical reconstruction of afferent and efferent pathways to the urinary bladder after conus medullaris injury in a rat model. $J$ Reconstr Microsurg. 2008; 24(8):575-581. 
7. Navarro $X$, Vivo $M$, Valero-Cabre A. Neural plasticity after peripheral nerve injury and regeneration. Prog Neurobiol. 2007;82:163-201.

8. Wang M, Li ZY, Xu WD, Hua XY, Xu JG, Gu YD. Sensory restoration in cortical level after a contralateral C7 nerve transfer to an injured arm in rats. Neurosurgery. 2010;67:136-143.

9. Fraser C, Power M, Hamdy S, Rothwell J, Hobday D, Hollander I, et al. Driving plasticity in human adult motor cortex is associated with improved motor function after brain injury. Neuron. 2002;34:831-840.

10. Fouad K, Pedersen V, Schwab ME, Brosamle C. Cervical sprouting of corticospinal fibers after thoracic spinal cord injury accompanies shifts in evoked motor responses. Curr Biol. 2001;11(22):1766-1770.

11. Ghosh A, Sydekum E, Haiss F, Peduzzi S, Zorner B, Schneider R, et al. Functional and anatomical reorganization of the sensory-motor cortex after incomplete spinal cord injury in adult rats. $J$ Neurosci. 2009;29(39):12210-12219.

12. Rosenzweig ES, Courtine G, Jindrich DL, Brock JH, Ferguson AR, Strand SC, et al. Extensive spontaneous plasticity of corticospinal projections after primate spinal cord injury. Nat Neurosci. 2010;13(12):1505-1510.

13. Schmidlin E, Wannier T, Bloch J, Rouiller EM. Progressive plastic changes in the hand representation of the primary motor cortex parallel incomplete recovery from a unilateral section of the corticospinal tract at cervical level in monkeys. Brain Res. 2004;1017(1-2):172-183.

14. Jurkiewicz MT, Crawley AP, Verrier MC, Fehlings MG, Mikulis DJ. Somatosensory cortical atrophy after spinal cord injury: a voxel-based morphometry study. Neurology. 2006;66:762-764.

15. Lee BH, Lee KH, Kim UJ. Injury in the spinal cord may produce cell death in the brain. Brain Res. 2004; 1020:37-44.

16. Wu J, Stoica BA, Luo T, Sabirzhanov B, Zhao Z, Guanciale K, et al. Isolated spinal cord contusion in rats induces chronic brain neuroinflammation, neurodegeneration, and cognitive impairment. Involvement of cell cycle activation. Cell Cycle. 2014;13(15):2446-2458.

17. Jure I, Pietranera L, De-Nicola AF, Labombarda F. Spinal Cord Injury Impairs Neurogenesis and Induces Glial Reactivity in the Hippocampus. Neurochem Res. 2017;42(8):2178-2190.

18. Shoham S, Halgren E, Maynard EM, Normann RA. Motor-cortical activity in tetraplegics. Nature. 2001;413(6858):793.

19. Jurkiewicz T, Mikulis DJ, Mcllroy WE, Fehlings MG, Verrier MC. Sensorimotor cortical plasticity during recovery following spinal cord injury: a longitudinal fMRI study. Neurorehabil Neural Repair. 2007;21(6): 527-38.

20. Jurkiewicz MT, Mikulis DJ, Fehlings MG, Verrier MC. Sensorimotor cortical activation in patients with cervical spinal cord injury with persisting paralysis. Neurorehabil Neural Repair. 2010;24(2):136-140.

21. Lotze M, Laubis-Herrmann U, Topka H. Combination of TMS and fMRI reveals a specific pattern of reorganization in M1 in patients after complete spinal cord injury. Restor Neurol Neurosci.2006, 24(2):97-107. 
22. Lou L, Shou T, Li Z, Li W, Gu Y. Transhemispheric functional reorganization of the motor cortex induced by the peripheral contralateral nerve transfer to the injured arm. Neuroscience. 2006;138:1225-1231.

\section{Figures}

a

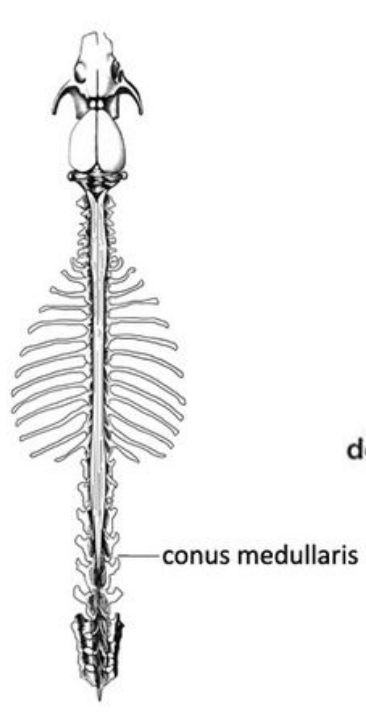

b

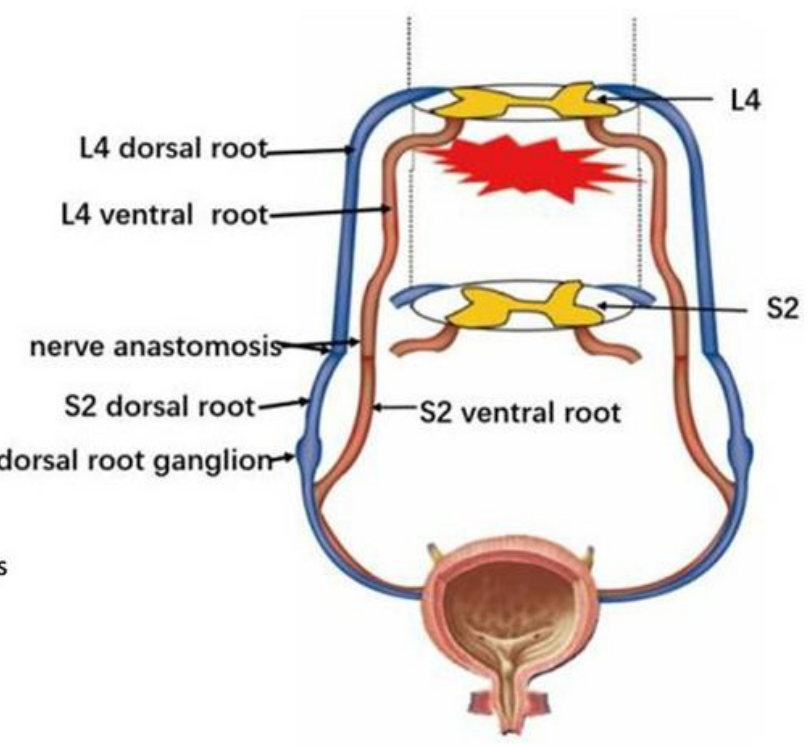

C

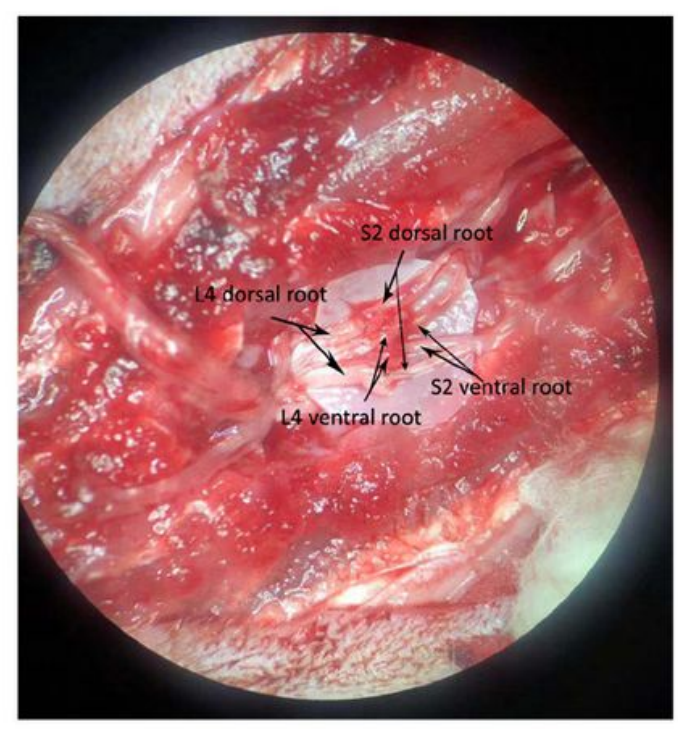

Figure 1

Mechanism of spinal cord injury and reconstruction of neural pathways a Schematic of rat spine: the conus medullaris was in level of L4. b Schematic of bladder function reconstruction surgery: the spinal cord and spinal nerves below L4 were cut, leading to blockade of bladder-cerebral nerve afferent and efferent pathways.the dorsal and ventral roots of bilateral S2 were anastomosed with the dorsal and ventral roots of bilateral L4. c Intraoperative picture of bladder function reconstruction surgery. 
a

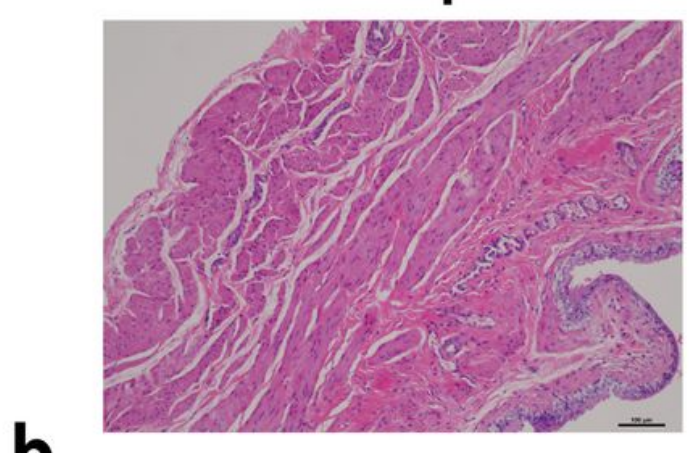

Group A

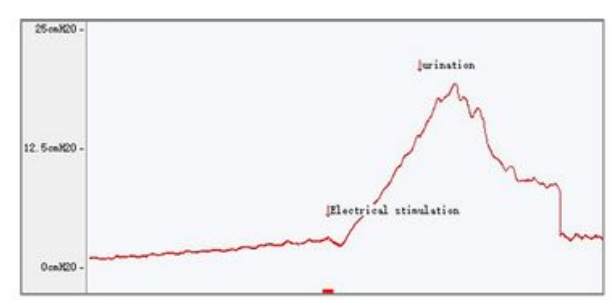

Group B
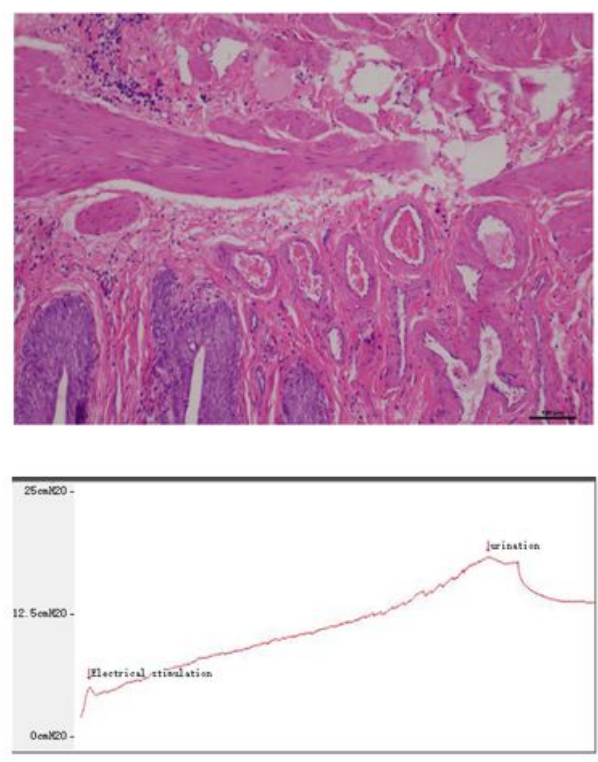

Group C
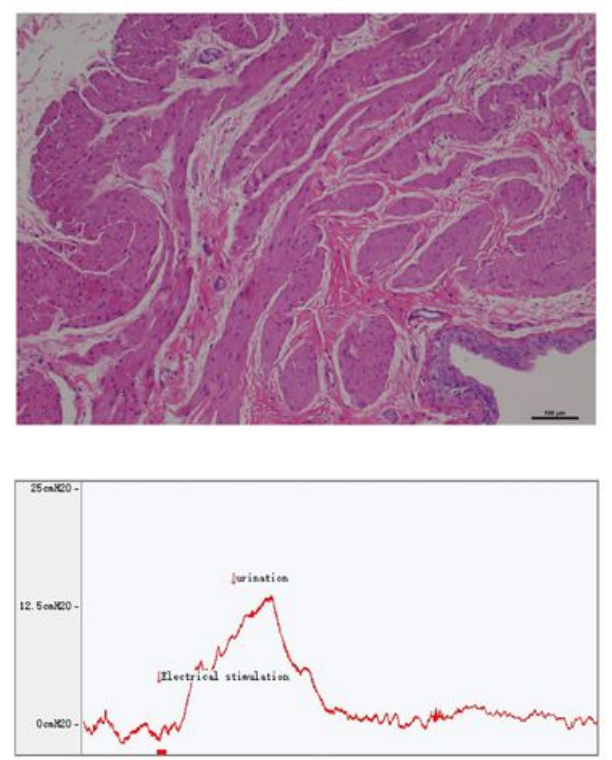

Figure 2

Morphological changes of the bladder and urodynamics at 3 months after surgery a The bladders of the three study groups were histologically analyzed by H\&E staining (x100). The bladder mucosa from rats in Group A appeared smooth and the muscle layer was slightly thickened. In rats from Group B, the bladder mucosa was rough and the mucosal cells appeared disordered. The muscle layer was thickened and hypertrophic with fibrous tissue proliferation and inflammatory cell infiltration.In rats from Group $\mathrm{C}$, the bladder mucosa was smooth and the muscle layer was thin, and the smooth muscle cells were small and arranged regularly. b Electrophysiological features of the three study groups. The electrophysiological results from rats in Groups $\mathrm{A}$ and $\mathrm{C}$ at 3 months after surgery showed after electrical stimulation the intravesical pressure fluctuated drastically. The intravesical pressure did not fluctuate in Group B. 


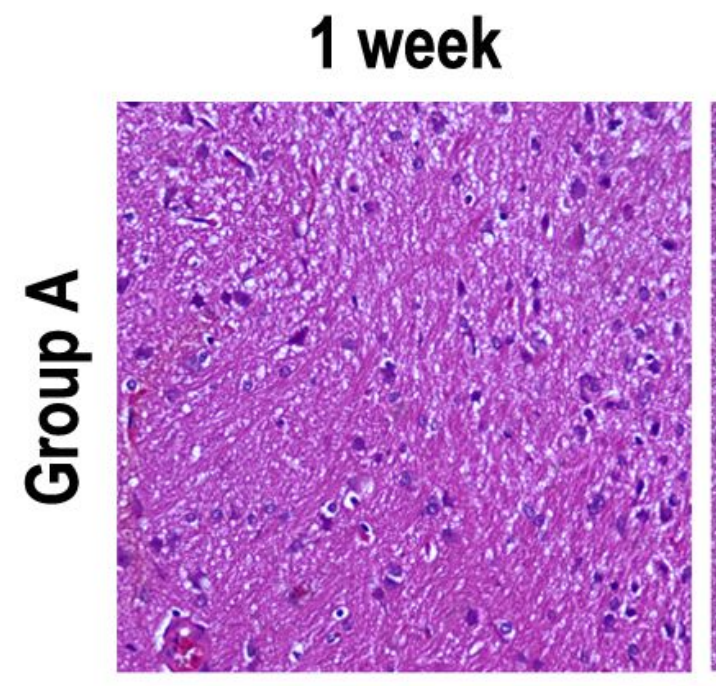

\section{1 month}
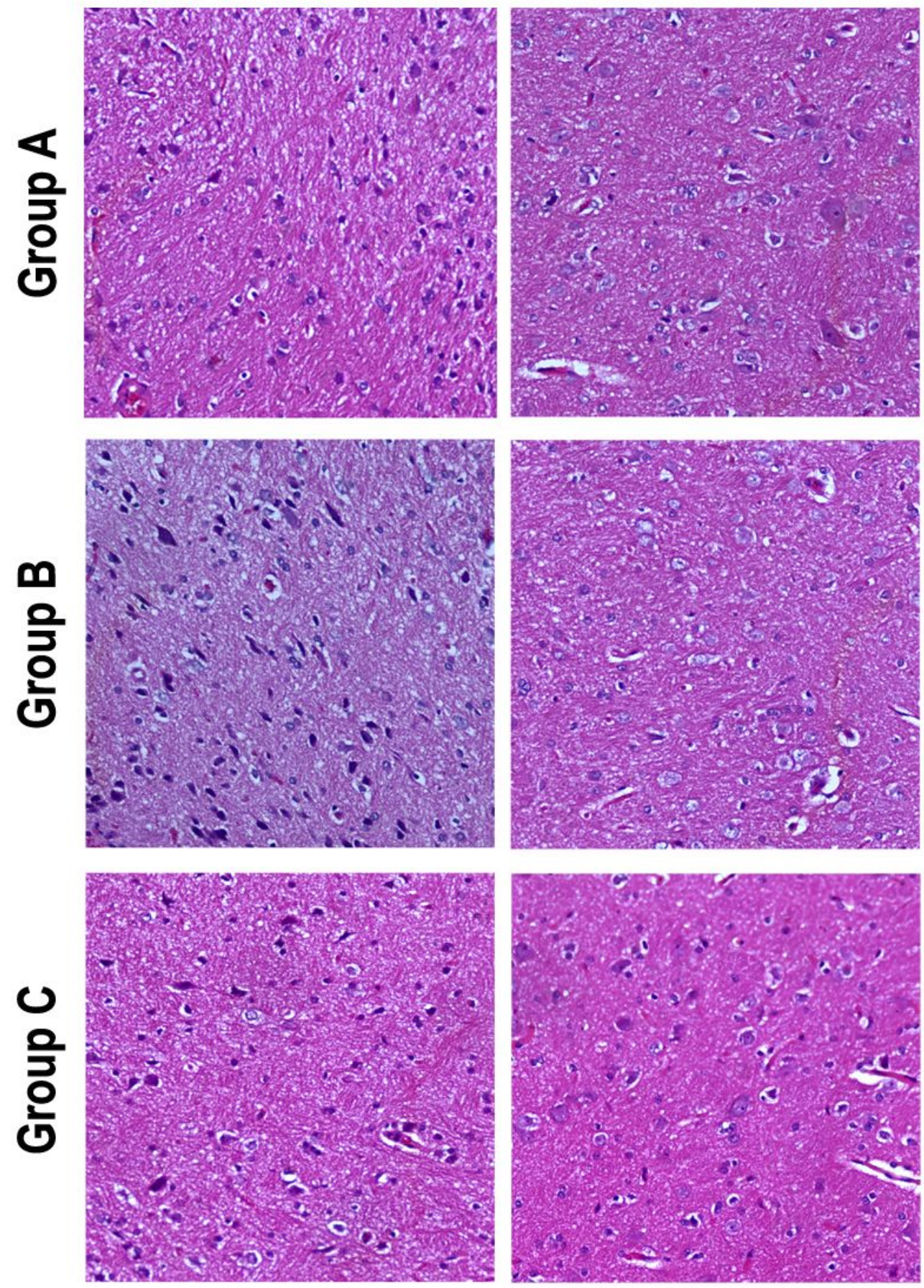
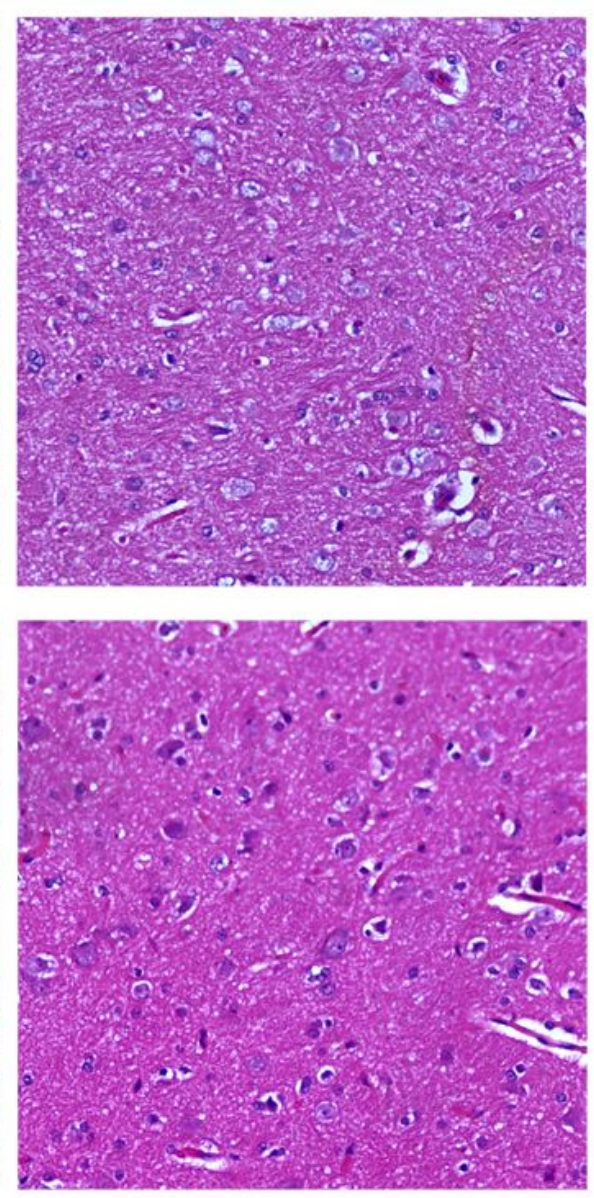

\section{3 months}
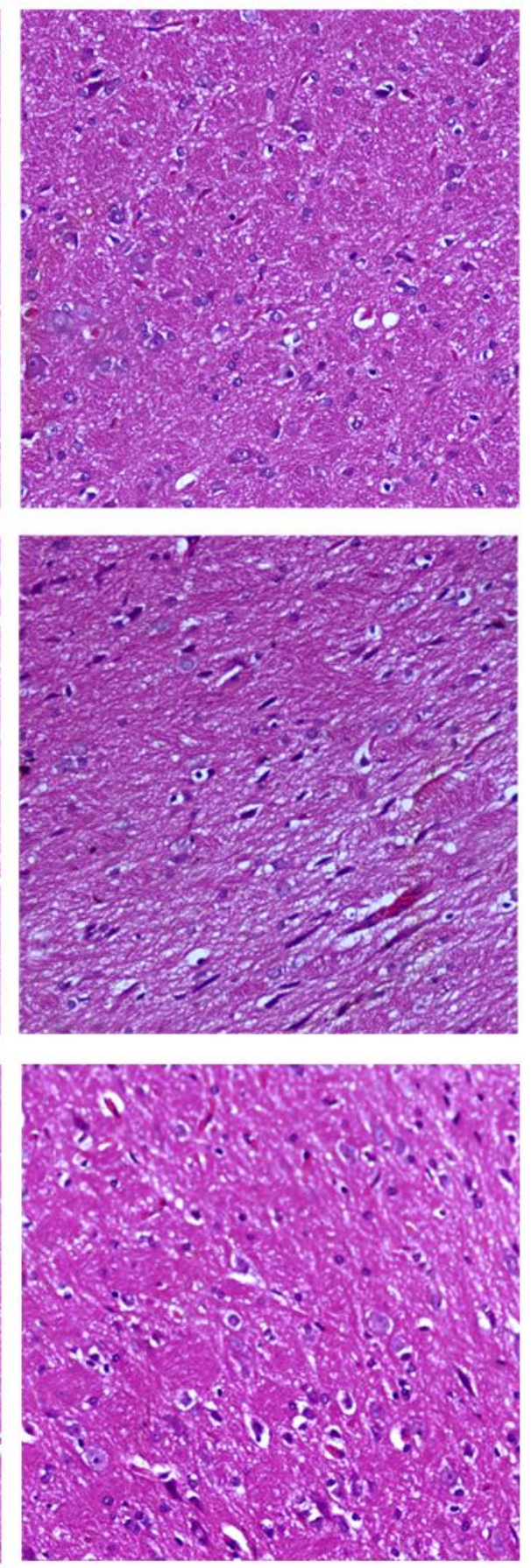

\section{Figure 3}

Pontine neurons in the three study groups, analyzed by H\&E staining There was no significant morphological difference in the pons between Groups A, B, and C at one day after surgery. One week after surgery, the extracellular space around the neurons was slightly increased in Groups A and B compared with that in Group C, and one month after surgery, some neurons showed nuclear pyknosis in Groups A and $B$. Three and six months after surgery, an accumulation of interstitial fibrous tissue was evident. 
a

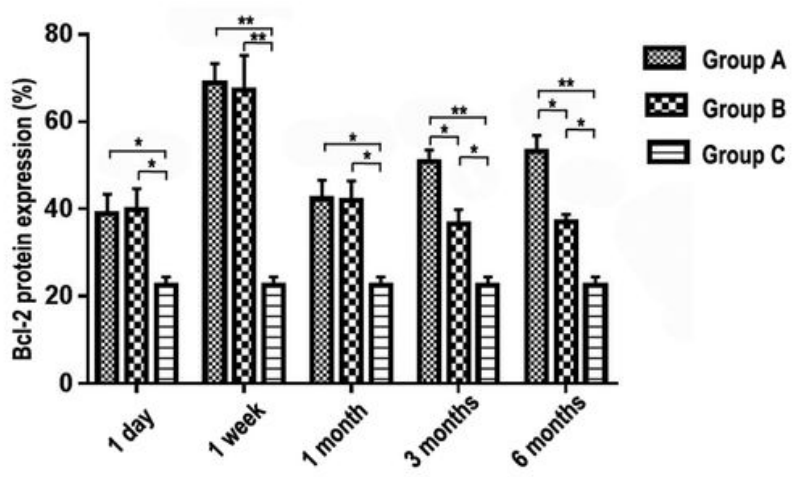

C

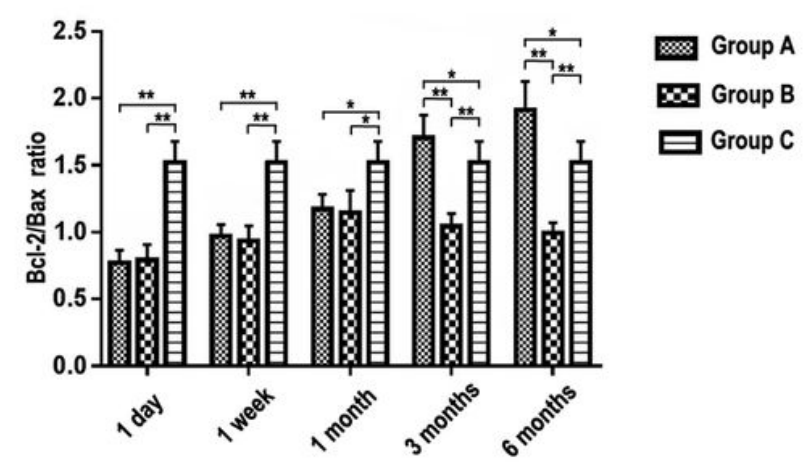

b

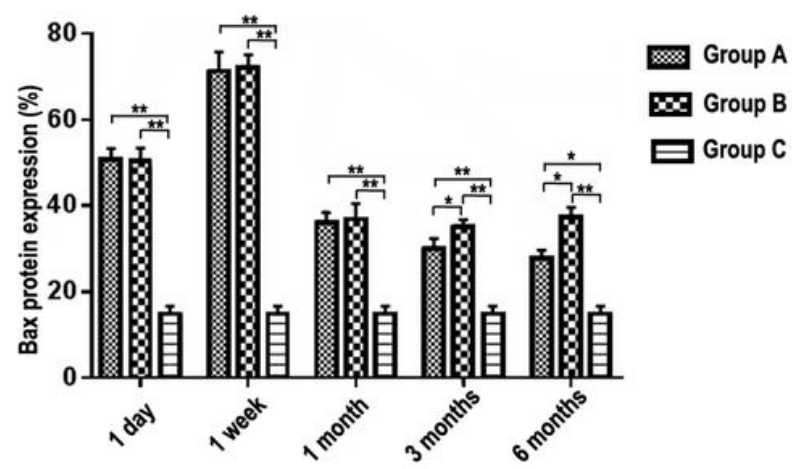

d

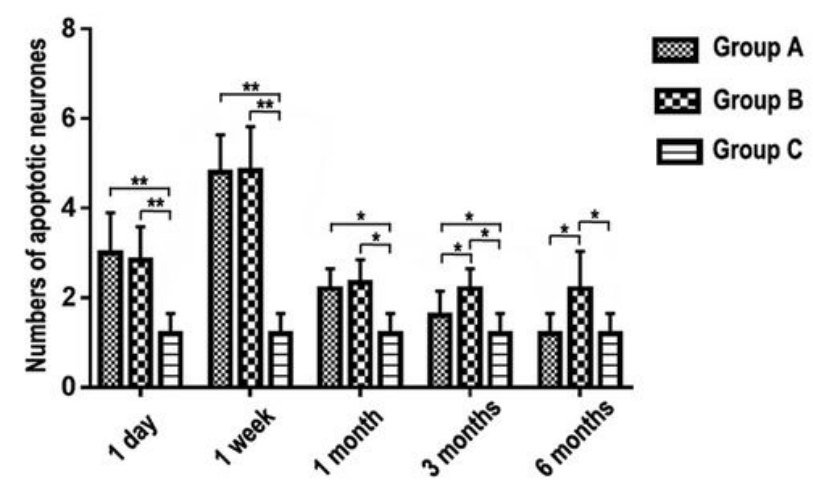

Figure 4

$\mathrm{Bcl}-2$, Bax protein expression in pontine neurons and number of apoptotic neurons of the three study groups at each timepoint a Fluorescence intensities of $\mathrm{Bcl}-2$ protein expression in (A) were quantified by gray value analysis. Data represent the means $\pm S D$. Two-way analysis of variance, ${ }^{*} P<0.05,{ }^{*} P<0.01$. $b$ Fluorescence intensities of Bax protein expression in $(A)$ were quantified by gray value analysis. Data represent the means \pm SD. Two-way analysis of variance, ${ }^{*} P<0.05,{ }^{*} \mathrm{P}<0.01$. c The Bcl-2/Bax ratios were measured by gray value analysis. Data represent the means \pm SD. Two-way analysis of variance, ${ }^{*} P$ $<0.05,{ }^{\star \star} P<0.01$. $d$ The number of apoptotic neurons were measured by gray value analysis. Data represent the means $\pm S D$. Two-way analysis of variance, ${ }^{*} P<0.05, * * P<0.01$. 


\section{1 week}
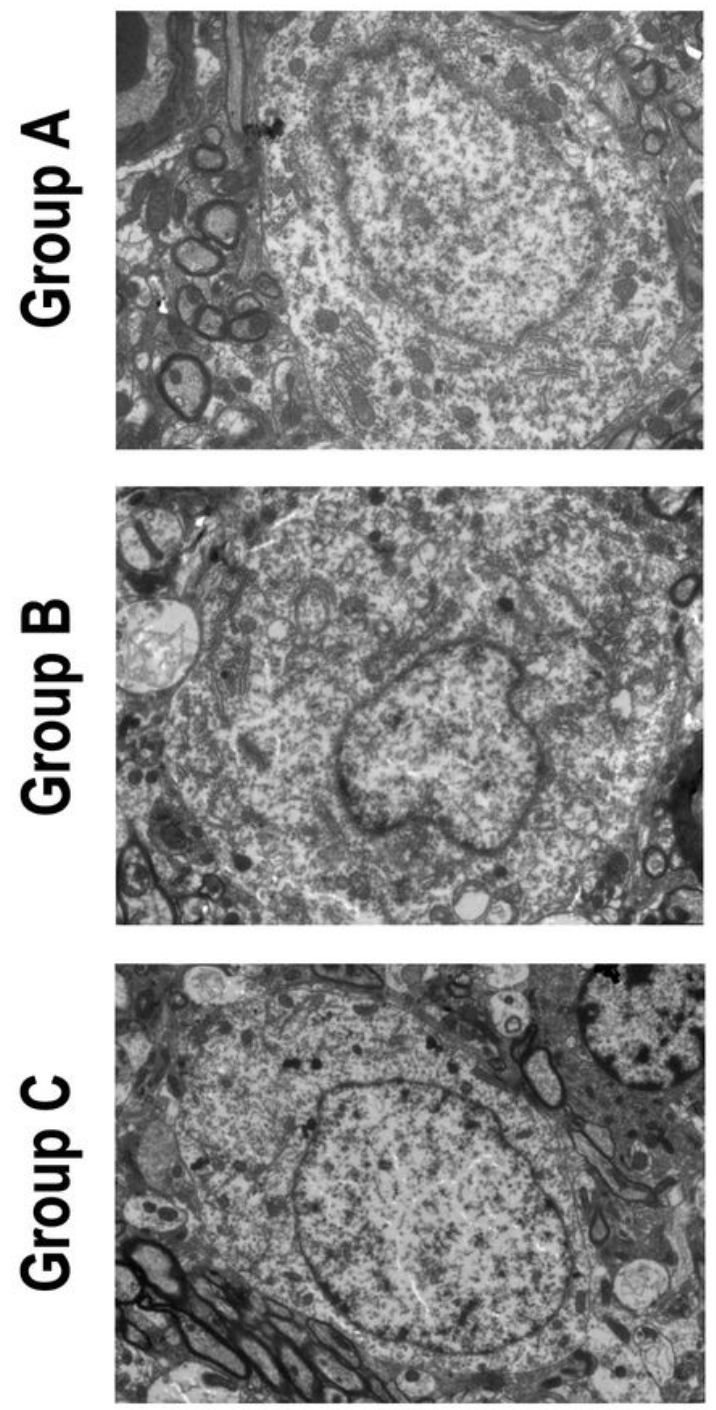

1 month
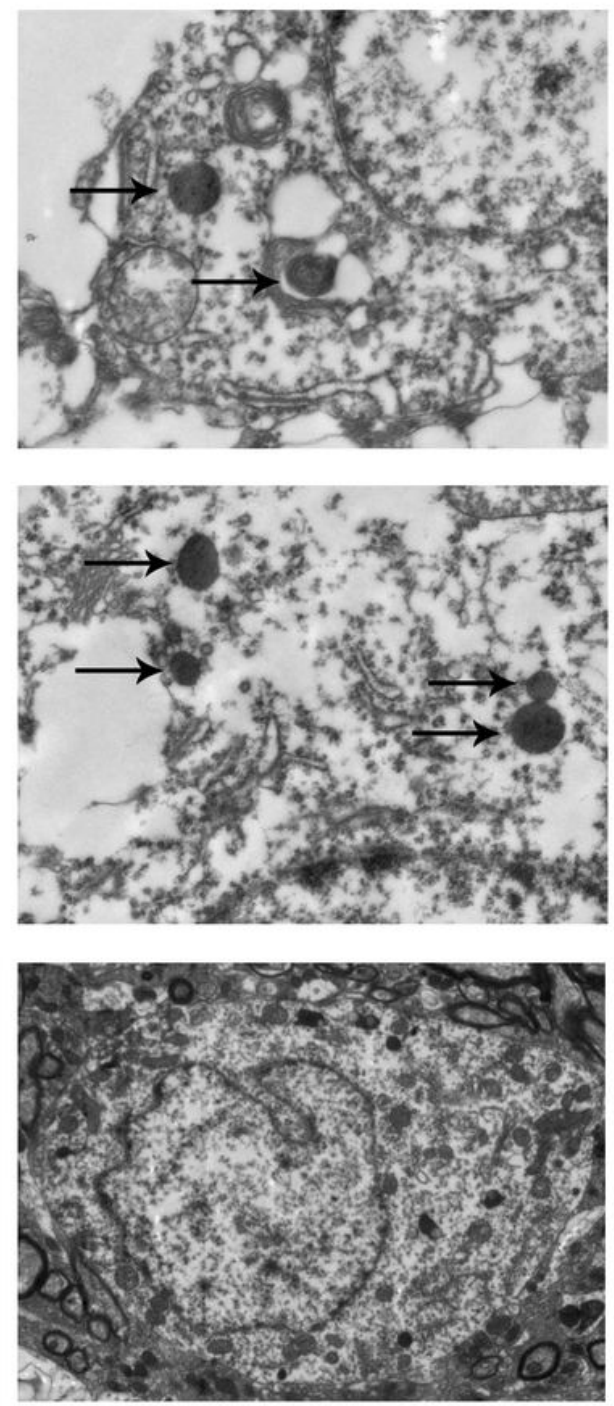

\section{3 months}
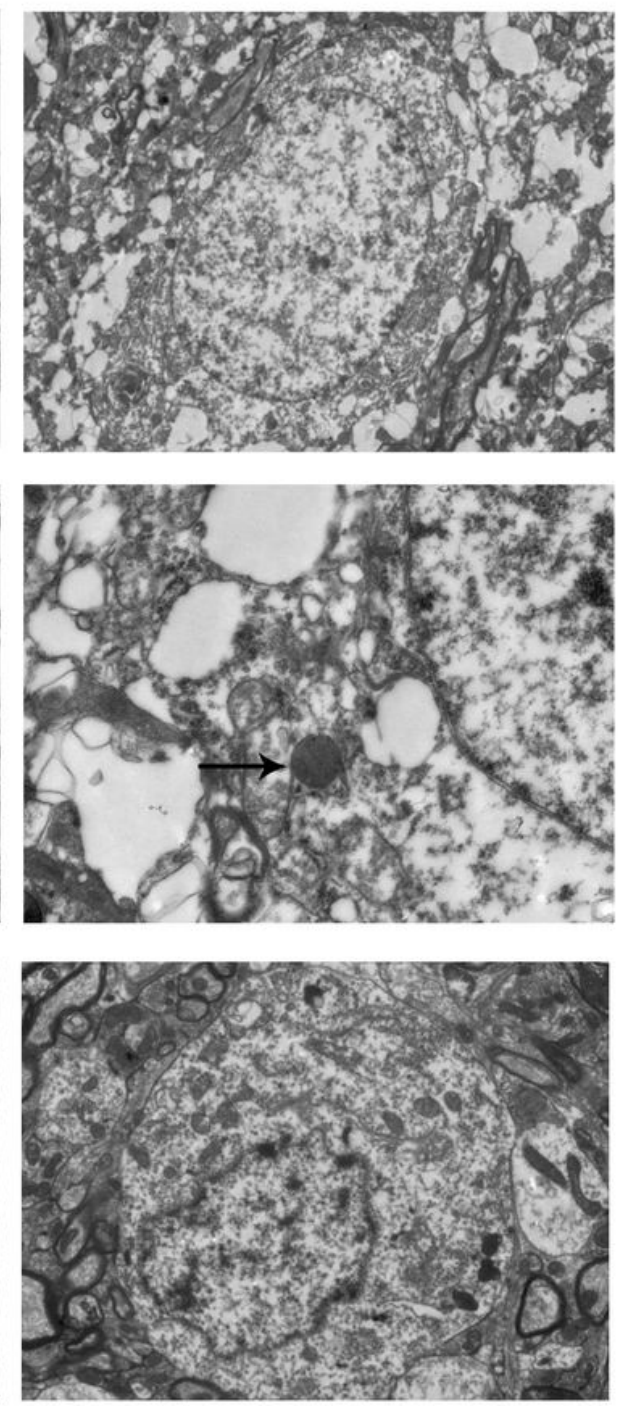

Figure 5

Pontine neurons of the three treatment groups, visualized by TEM (x2000) TEM analysis showed nuclear pyknosis and mitochondrial vacuolization in Groups A and B at one month after surgery, as well as pyknotic margination of chromatin, intercellular space enlargement, and apoptotic bodies. The number of apoptotic cells increased in Group B. Arrows indicate apoptotic bodies.

\section{Supplementary Files}

This is a list of supplementary files associated with this preprint. Click to download.

- NC3RsARRIVEGuidelinesChecklistfillable.pdf 